\title{
Discrete lattice symmetry breaking in a two-dimensional frustrated spin-1 Heisenberg model
}

\author{
Ji-Yao Chen, Sylvain Capponi, and Didier Poilblanc \\ Laboratoire de Physique Théorique, IRSAMC, Université de Toulouse, CNRS, UPS, 31062 Toulouse, France
}

(Received 8 April 2018; published 3 July 2018)

\begin{abstract}
Spontaneous discrete symmetry breaking can be described in the framework of projected entangled pair states (PEPS) by linearly superposing local tensors belonging to two (or more) symmetry classes of tensors. This is illustrated in the case of a frustrated spin-1 Heisenberg model on the square lattice, which hosts a nematic spin liquid spontaneously breaking lattice $\pi / 2$-rotation symmetry. A superposition of SU(2)-symmetric PEPS tensors belonging to two irreducible representations of the lattice point group is shown to capture accurately the properties of the nematic phase, as shown from a comparison to exact diagonalizations and density matrix renormalization group results.
\end{abstract}

DOI: 10.1103/PhysRevB.98.045106

\section{INTRODUCTION AND MODEL}

The Ginzburg-Landau (GL) paradigm offers a general and elegant framework to describe low-energy phases in condensed-matter systems in terms of spontaneous symmetry breaking. For example, magnetic ordering in quantum spin systems can be viewed as a spontaneous breaking of the underlying spin rotation $(\mathrm{SU}(2))$ symmetry. Electronic charge-density waves or (spin) valence-bond crystals spontaneously break the underlying lattice translation symmetry. More interestingly, in the nematic electronic phases of some pnictides [1,2] (a class of iron-based high- $T_{c}$ superconductors) orientational order sets up that resembles - to some extent-classical liquid crystals of molecules [3]. In the electronic system, the lattice (discrete) point-group symmetry is spontaneously broken, a scenario fitting well into the GL scheme. E.g., on the two-dimensional square lattice, of point group $C_{4 v}$, the horizontal $(x)$ and vertical $(y)$ axes become nonequivalent, so that transport or correlations along these two directions become different.

Our aim here is to investigate such a phenomenon in simple quantum spin magnets. In such a case, the nematic phase can be viewed as a melted (thermal [4,5] or quantum [2]) magnetic stripe phase (of magnetic wave-vector $\mathbf{q}=(\pi, 0)$ or $(0, \pi))$ where the $\mathrm{SU}(2)$ spin symmetry has been restored while orientational order (spontaneous breaking of the 90-degree lattice rotation) still persists. Therefore, nematic phases could potentially appear in the immediate proximity of magnetic stripe phases. Alternatively, nematic phases can also emerge from the Néel phase via a proliferation of monopoles [6]. Monopoles carry Berry phases [7] implying that, in the phase where the monopoles proliferate with spin $S=1$ (or oddinteger spin), there is nematic order [8].

To be more specific, let us consider the frustrated spin-1 Heisenberg model on the two-dimensional square lattice,

$$
\begin{aligned}
H= & J_{1} \sum_{\langle i, j\rangle} \mathbf{S}_{i} \cdot \mathbf{S}_{j}+J_{2} \sum_{\langle k, l\rangle\rangle} \mathbf{S}_{k} \cdot \mathbf{S}_{l} \\
& +K_{1} \sum_{\langle i, j\rangle}\left(\mathbf{S}_{i} \cdot \mathbf{S}_{j}\right)^{2},
\end{aligned}
$$

where the first and third sums are taken over nearest-neighbor (NN) bonds and the second sum runs over next-nearestneighbor (NNN) bonds. For simplicity, we set the NN and NNN bilinear couplings to $J_{1}=1$ and $J_{2}=0.54$, corresponding approximately to the maximally frustrated regime [9], and vary the biquadratic coupling $K_{1}$. As shown in Ref. [9], the $J_{1}-J_{2}$ model (with spatial anisotropy of the $J_{1}$ coupling) may host a quantum-disordered phase, in a narrow region of parameter space, between a Néel phase and a (magnetic) stripe phase. We argue in this paper that the quantum-disorder phase spontaneously breaks lattice rotation symmetry, being of nematic character. For our choice of the bilinear couplings the nematic phase is stable in the range $0 \lesssim K_{1} \lesssim 0.15$.

Our strategy to explore the physics of the above model is to combine different numerical techniques, such as exact diagonalizations (ED), density matrix renormalization group (DMRG), and tensor network methods [10-14]. In particular, we shall focus on $\mathrm{SU}(2)$-symmetric projected entangled pair states (PEPS) to describe the nematic spin liquid phase. More precisely, we construct an explicit PEPS wave function that provides a faithful representation of the symmetry-broken nonmagnetic state directly in the thermodynamic limit. In contrast to usual PEPS calculations, which approach the ground state (GS) of the model via imaginary time evolution (starting from some initial random state), we use a more elegant framework. As is commonly known, the manifold of PEPS is not simply connected but has many different phases, which makes the search for spin liquid phases in frustrated models challenging. It is therefore appropriate, following the pioneering work in Ref. [15], to study each class separately-with a clear understanding of the physical nature of the variational wave function - using a variational optimization scheme.

\section{LANCZOS EXACT DIAGONALIZATIONS}

As a preliminary study to explore the physics of Hamiltonian (1), we have performed Lanczos ED of finite periodic $4 \times 4$ and $\sqrt{20} \times \sqrt{20}$ clusters (topologically equivalent to a torus). "Level spectroscopy" is a useful tool to get insights on the nature of the $(T=0)$ quantum phase. In genuine magnetically 

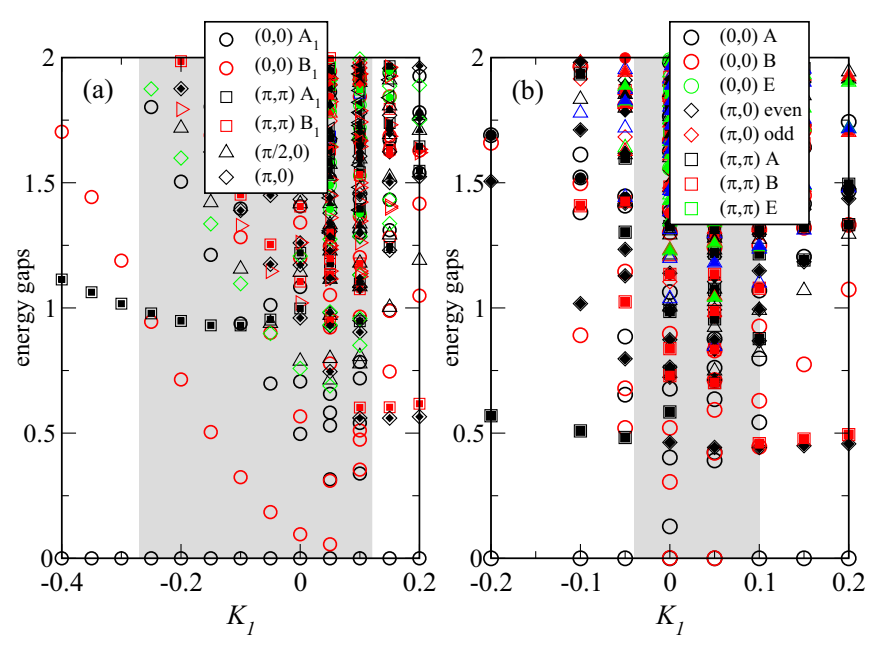

FIG. 1. Low-energy spectra of the Heisenberg Hamiltonian (1) vs $K_{1}$ obtained by Lanczos ED of periodic (a) $4 \times 4$ and (b) $\sqrt{20} \times \sqrt{20}$ clusters. The different symbols (colors) correspond to different momenta (IRREP of the lattice point group). Open (filled) symbols correspond to singlet (triplet) states. The shaded/white regions are characterized by the singlet/triplet nature of the first excitation above the GS, suggesting possible nematic/magnetic phases (see text).

ordered phases (spontaneously breaking the continuous SU(2) spin-rotation symmetry) one expects low-energy triplets (socalled Anderson's tower of states) collapsing onto the (singlet) GS in the thermodynamic limit. In contrast, in the case of a spin liquid only breaking a discrete space-group symmetry, one expects a finite number of quasi-degenerate GS separated from the rest of the spectrum by a finite gap. In addition, the quantum numbers associated with the quasi-degenerate GS give insights on the precise nature of the symmetry-broken phase.

Figure 1 shows a narrow region of the parameter $K_{1}$ where the two lowest eigenstates are momentum $\mathbf{q}=(0,0)$ singlets belonging to two different $A$ and $B$ irreducible representations (IRREPs) of the point group. Note that, although the 20-site cluster does not possess all the square lattice symmetries (its point group is $C_{4}$ instead of $C_{4 v}$ for the 16-site cluster and the infinite lattice) it nevertheless possesses the 90 -degree rotation that enables to distinguish between the $A$ and $B$ IRREPs. In particular, for $K_{1}=0.05$ the energy separation between the two states is very small (even not visible in Fig. 1(b)), and a significant gap appears above. This low-energy spectrum is typical of a lattice nematic phase with nonequivalent correlations in the $x$ and $y$-axis directions. In the neighboring left and right regions the first excitation is a triplet state, suggesting the occurrence of magnetic phases. The momentum $\mathbf{q}=(\pi, \pi)(\mathbf{q}=(\pi, 0)$ and $(0, \pi))$ of the first triplet excited state is compatible with Néel (stripe) magnetic order. Note, however, that the extension of the supposed nematic spin liquid phase strongly depends on the cluster, so that complementary methods are required to ascertain its stability.

\section{DMRG PHASE DIAGRAM}

To better characterize the three phases at play, we have performed DMRG simulations on $L \times W$ cylinders, using typically $L=2 W$ (with periodic boundary condition in the shorter
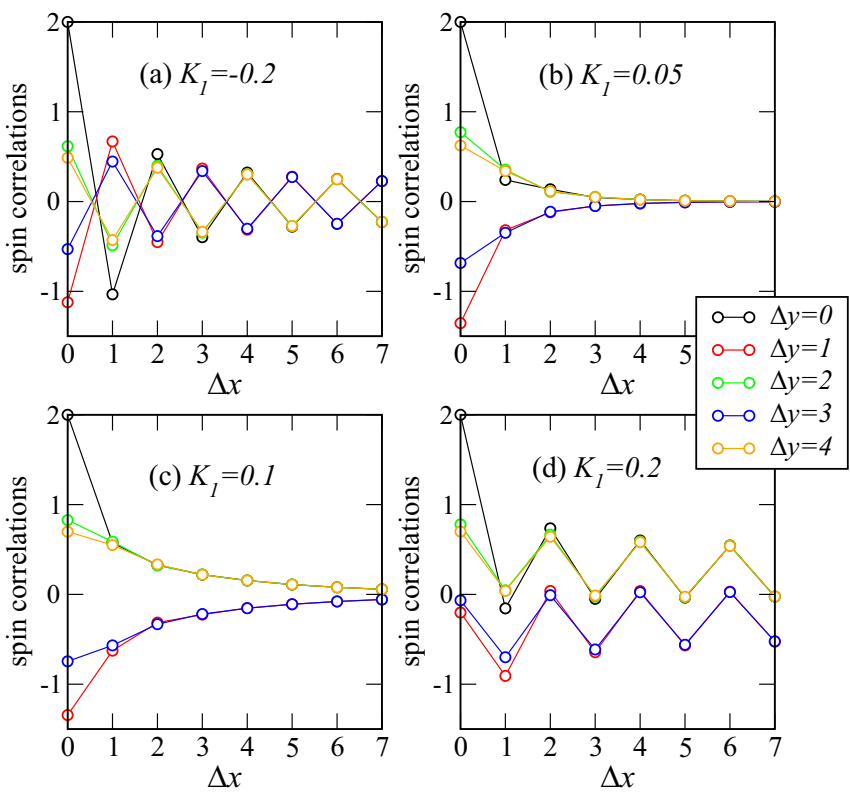

FIG. 2. DMRG results for spin-spin correlations (3) at distance $(\Delta x, \Delta y)$ obtained on a $16 \times 8$ cylinder for various $K_{1}$ indicated on the plot. Data are averaged in the middle $8 \times 8$ region to reduce finite-size effects.

direction). In order to characterize the putative phases, we have measured their respective order parameters. For nematicity, we directly measure the $x$ and $y$ bilinear bond energies in the bulk of the system [16] and define an order parameter as

$$
\mathrm{OP}=\left|\left\langle\mathbf{S}_{x, y} \cdot \mathbf{S}_{x+1, y}-\mathbf{S}_{x, y} \cdot \mathbf{S}_{x, y+1}\right\rangle\right| .
$$

Note that since our cylinder explicitly breaks lattice rotation symmetry, we can obtain a finite value even on a finite cluster. But we do need to perform finite-size scaling to check its thermodynamic limit value, see below and Appendix A. For magnetic phases (Néel or stripe), since our finite cluster cannot break SU(2) symmetry, we need to compute relevant spin-spin correlations:

$$
C(\Delta x, \Delta y)=\left\langle\mathbf{S}_{x, y} \cdot \mathbf{S}_{x+\Delta x, y+\Delta y}\right\rangle .
$$

In Fig. 2, we plot some examples of real-space spin-spin correlations for various $K_{1}$. It is already apparent that the modulation changes from $(\pi, \pi)$ wavevector for negative $K_{1}$ values to $(0, \pi)$ for positive ones. Note also that, since we are only using U(1) symmetry, our DMRG simulation can end up in a state with a finite local $\left\langle S_{i}^{z}\right\rangle$ (for instance, we measure $\left|\left\langle S_{i}^{z}\right\rangle\right| \sim 0.5$ when $K_{1}= \pm 0.2$ ), which suggests long-range magnetic order in the thermodynamic limit. Note however that, for intermediate values $K_{1}=0.05$ and $K_{1}=0.1$, there are no oscillations and correlations become very small at the largest available distances.

In order to be more quantitative, we have chosen to measure all spin correlations within a $W \times W$ subset in the center of each $2 W \times W$ cylinder in order to compute the structure factors $m^{2}(\pi, \pi)$ and $m^{2}(\pi, 0)$ respectively:

$$
m^{2}(\mathbf{q})=\frac{1}{N_{s}} \sum_{i, j \in \mathcal{C}}\left\langle\mathbf{S}_{i} \cdot \mathbf{S}_{j}\right\rangle e^{i \mathbf{q} \cdot \mathbf{r}}
$$




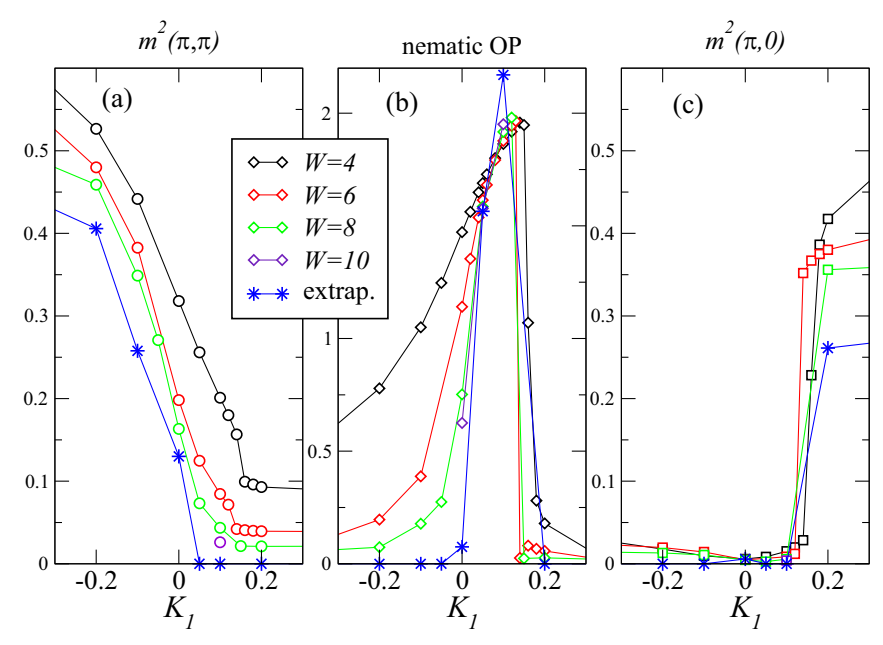

FIG. 3. DMRG results vs $K_{1}$ obtained on various $2 W \times W$ cylinders (with open boundary conditions in the long direction, see text): (a) Square of the staggered magnetization (4); (b) nematic order parameter (2); (c) square of the $\mathbf{q}=(\pi, 0)$ magnetic structure factor (4). Extrapolation leads to a phase diagram with three successive phases: Néel, nematic, and magnetic stripe phase, respectively, when increasing $K_{1}$ parameter.

where the sum runs over all sites $(i, j)$ within the central part with $N_{s}=W^{2}$ sites and $\mathbf{r}$ is the relative distance between $i$ and $j$. While a cylinder geometry favors a modulation with wavevector $(0, \pi)$ for positive $K_{1}$ (see Fig. 2(d)), we rather consider here the structure factor at $\mathbf{q}=(\pi, 0)$ that should also converge to the square of the order parameter in the thermodynamic limit and has less finite-size effects, see Appendix A.

In Fig. 3, we summarize our DMRG results plotting, side by side, the order parameters of the three phases as a function of $K_{1}$. Our data are compatible with the existence and stability of three phases: two magnetically ordered ones (Néel for $K_{1} \lesssim 0$ and stripe for $K_{1} \gtrsim 0.15$ ) and a nonmagnetic nematic phase in between [17]. Moreover, we observe rather sharp transitions (especially at positive $K_{1}$ ) so that presumably both quantum phase transitions are first order, as expected from a standard Ginzburg-Landau analysis.

\section{PEPS ANSATZ FOR THE NEMATIC PHASE}

Both Lanczos ED and DMRG results suggest that, in the range $0<K_{1}<0.15$, there is no magnetic order. This motivates us to study this region with $\mathrm{SU}(2)$-symmetric PEPS directly in the thermodynamic limit. To do so, we first apply a $\pi$ rotation along the $Y$-spin axis on one of the sublattices, and then approximate the GS wave function(s) in terms of a unique site tensor $\mathcal{A}_{\alpha \beta \gamma \delta}^{s}$, where the greek indices label the states of the $D$-dimensional virtual space $\mathcal{V}$ attached to each site in the $z=4$ directions of the lattice, and $s=0, \pm 1$ is the $S_{z}$ component of the physical spin- 1 . A (lattice) nematic state bears symmetry properties that greatly constrain the PEPS ansatz. To construct such an ansatz, we use a classification of fully SU(2)-symmetric (i.e., singlet) PEPS proposed recently [18] in terms of the four virtual composite spins attached to each site and the IRREP of the square lattice point-group $C_{4 v}$.

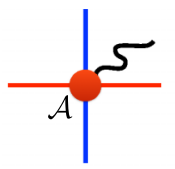

(a)

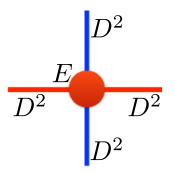

(b)

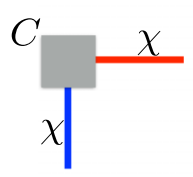

(d)

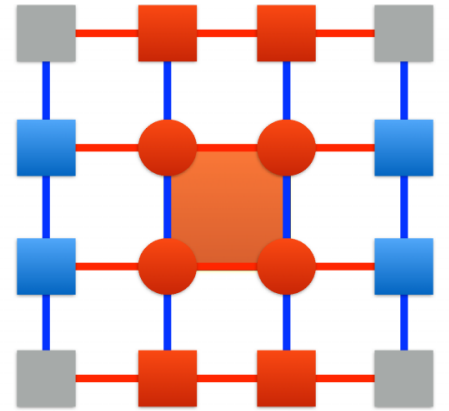

(c)

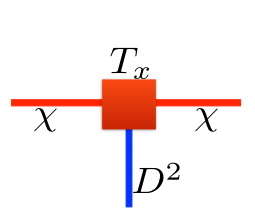

(e)

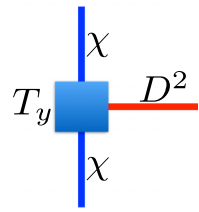

(f)

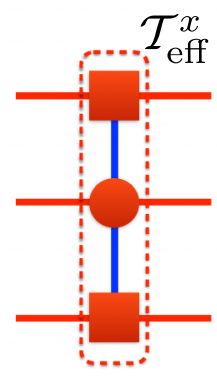

(g)

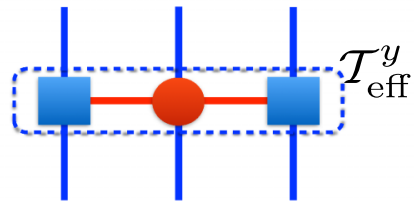

(h)
FIG. 4. CTM RG for nematic PEPS with one-site unit cell. The $\pi / 2$ lattice rotation symmetry breaking is indicated by red/blue line in horizontal/vertical direction. (a) Nematic PEPS tensor $\mathcal{A}$. (b) Double tensor $E$ obtained by tracing out physical indices $E=\sum_{s} \overline{\mathcal{A}}^{s} \mathcal{A}^{s}$, where $\overline{\mathcal{A}}^{s}$ is complex conjugate of $\mathcal{A}^{s}$. (c) CTM RG environment for $2 \times 2$ cluster, constructed with corner matrix $C$, boundary tensor $T_{x, y}$ in horizontal/vertical direction, as shown separately in (d)-(f). The environment bond dimension is chosen to be $\chi=k D^{2}\left(k \in \mathbb{N}_{+}\right)$. Energy density is calculated by inserting either identity operator or the local Hamiltonian operator in the red shaded $2 \times 2$ cluster. (g), (h) is effective transfer matrix $\mathcal{T}_{\text {eff }}^{x, y}$ in horizontal/vertical direction, constructed with $T_{x, y}$ and $E$ tensor. The maximal correlation length in horizontal/vertical direction can be obtained from the largest two eigenvalues of $\mathcal{T}_{\text {eff }}^{x, y}$.

In this setting, the virtual space $\mathcal{V}$ is a direct sum of $\mathrm{SU}(2)$ IRREPs. Since the nematic state breaks only the 90-degree lattice rotation (but is invariant under axis reflection), the simplest adequate PEPS site tensors have the form

$$
\mathcal{A}=\mathcal{A}_{1}+\mathcal{B}_{1}=\sum_{a=1}^{N_{A}} \lambda_{a} \mathcal{A}_{1}^{a}+\sum_{b=1}^{N_{B}} \mu_{b} \mathcal{B}_{1}^{b},
$$

graphically shown in Fig. 4(a), where the real elementary tensors $\mathcal{A}_{1}^{a}$ and $\mathcal{B}_{1}^{b}$ have the same set of virtual spins and transform according to the $A_{1}$ (i.e., fully symmetric or $s$-wave like) and $B_{1}$ (i.e., $d$-wave like) IRREP. $\lambda_{a}$ and $\mu_{b}$ are the corresponding coefficients, chosen to be real, and $N_{A, B}$ is the number of such elementary tensors in each class. By reversing the overall sign 
TABLE I. Numbers of independent SU(2)-symmetric spin-1 tensors for the four different virtual spaces we consider, $D \leqslant 6$. The third (fourth) column gives the number of $\mathcal{A}_{1}\left(\mathcal{B}_{1}\right)$ tensors, and the last column is for the total number of tensors in the PEPS ansatz of the spin-1 nematic state.

\begin{tabular}{llrrc}
\hline \hline $\mathcal{V}$ & $D$ & $\mathcal{A}_{1}$ & $\mathcal{B}_{1}$ & Total number \\
\hline$\frac{1}{2} \oplus 0$ & 3 & 2 & 2 & 4 \\
$\frac{1}{2} \oplus 0 \oplus 0$ & 4 & 6 & 5 & 11 \\
$\frac{1}{2} \oplus \frac{1}{2} \oplus 0$ & 5 & 12 & 13 & 25 \\
$1 \oplus \frac{1}{2} \oplus 0$ & 6 & 13 & 13 & 26 \\
\hline \hline
\end{tabular}

of the coefficients $\mu_{b}$, the other degenerate GS can be obtained [19]. These tensors have been tabulated in Ref. [18] for $D \leqslant 6$, and their numbers for all virtual spaces $\mathcal{V}$ with good variational energy for the frustrated spin-1 Heisenberg model are listed in Table I. Following previous studies of the non-chiral or chiral frustrated spin- $1 / 2$ antiferromagnetic Heisenberg model (AFHM) [20,21], we consider a general superposition of all tensors of each class, and the coefficients $\lambda_{a}, \mu_{b}$ are considered as variational parameters. To further support the existence of a nematic spin liquid phase, we find it is interesting to consider, for comparison, the subclass of fully symmetric $\mathcal{A}_{1}$ PEPS constructed from the $\mathcal{A}_{1}^{a}$ tensors only, i.e., setting $\mu_{b}=0$ in Eq. (5). The subclass of $\mathcal{B}_{1}$ PEPS constructed with only $\mathcal{B}_{1}^{b}$ tensors, although giving also fully symmetric states, does not provide good energies (see text below) and therefore is not considered here.

To explore the physical properties, i.e., energy density and other observables, directly in the thermodynamic limit using infinite-PEPS (iPEPS), we apply corner transfer matrix (CTM) renormalization group (RG) techniques [22-25], taking advantage of simplifications introduced by the use of point-group symmetric tensors [20]. At each RG step a truncation of the CTM is performed by keeping (at most) $\chi$ eigenvalues/singular values and preserving exactly the SU(2) multiplet structure. For a fully symmetric $\mathcal{A}_{1}$ PEPS (given by all $\mu_{b}=0$ in Eq. (5)) the CTM is Hermitian, and truncation can be done using ED, following Ref. [20]. For a genuine nematic state given by Eq. (5), the CTM is not Hermitian due to lattice rotation symmetry breaking, and we need to use a singular value decomposition instead. The specific environment tensors for our nematic PEPS, obtained from CTM RG, are shown in Figs. 4(c)-4(f), which include the corner matrix $C$ and the boundary tensors $T_{x, y}$ in the horizontal/vertical direction. For the fully symmetric $\mathcal{A}_{1}$ PEPS, the boundary tensors in both directions are actually the same [20]. To optimize the coefficients in Eq. (5) [26-28], we perform a conjugate gradient method [29], starting with small $\chi$ and gradually increasing $\chi$ up to a maximum $\chi=\chi_{\text {opt }}$. In this process, we take $\chi=k D^{2}\left(k \in \mathbb{N}_{+}\right)$, and, since the number of elementary tensors in each class is small (see Table I), we are able to calculate the gradient using a simple finite difference method. After optimization, we continue to perform CTM RG with several larger $\chi$ values to obtain physical observables and then, eventually, take the limit $\chi \rightarrow \infty$ (using a "rigid" ansatz) by extrapolating the data.

Let us now first present results for the energy density at $K_{1}=0,0.05,0.1$. For all these cases, we shall use the full $\mathcal{A}_{1}+$
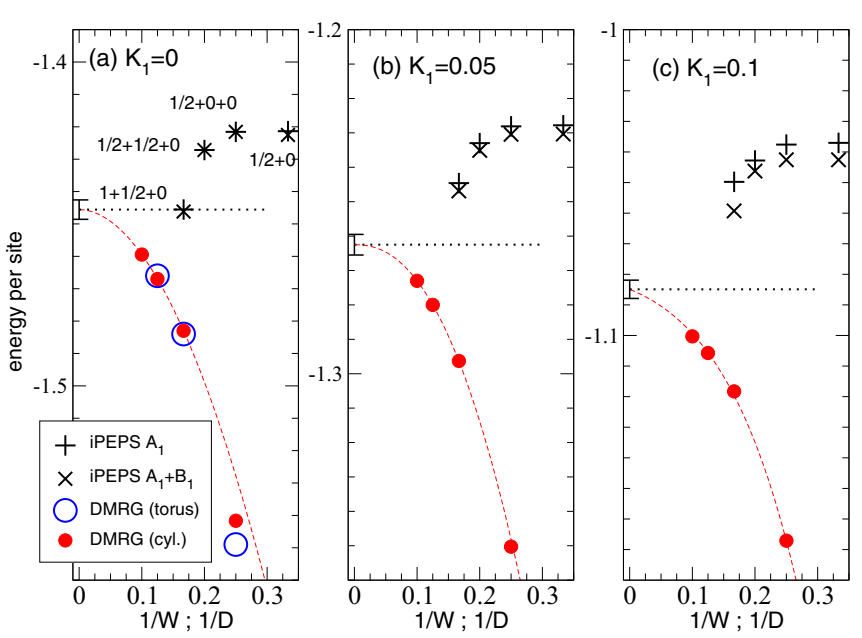

FIG. 5. iPEPS variational energies per site (extrapolated in the limit $\chi \rightarrow \infty$ ) vs $1 / D$. A comparison to DMRG data obtained on $2 W \times W$ cylinders (see text) and plotted vs $1 / W$ is shown. In (a), data for $W \times W$ tori [9] are also shown.

$\mathcal{B}_{1}$ ansatz of Eq. (5) as well as the restricted $\mathcal{A}_{1}$ symmetric ansatz. We have explored all different classes of nematic states with $D \leqslant 6$, and focus here on the virtual spaces $\mathcal{V}=\frac{1}{2} \oplus$ $0, \frac{1}{2} \oplus 0 \oplus 0, \frac{1}{2} \oplus \frac{1}{2} \oplus 0,1 \oplus \frac{1}{2} \oplus 0$, corresponding to $D=$ 3-6, respectively, with which the best variational energies have been found for each given value of $D$ (and $K_{1}=0$ ). The energies for each class are plotted as a function of $D^{2} / \chi$ (see Appendix B) and extrapolated to $\chi=\infty$. The extrapolated PEPS energies are then compared to DMRG results in Fig. 5 showing nice agreement, in particular for $K_{1}=0$. This indicates our ansatz provides a good approximation for the true ground state in the thermodynamic limit, albeit with relatively small bond dimension $D$.

The fully symmetric $\mathcal{A}_{1}$ state with $D=3$ corresponds in fact to the so-called resonating Affleck-Kennedy-Lieb-Tasaki loop (RAL) state [30], and the larger $D$ case can be viewed as certain extensions of the RAL state. The nematic state is then obtained by mixing with $\mathcal{B}_{1}$ components and therefore breaking the lattice rotation symmetry. It is interesting to note that, even with $D=3$, i.e., the RAL state, the energy is already fairly good, considering the fact that there is only one variational parameter to play with in the fully symmetric case. This is quite similar to the spin- $1 / 2$ case, where a one-parameter family of long-range resonating valence bond (RVB) states gives also a good ansatz for the spin-1/2 $J_{1}-J_{2}$ AFHM [20]. Starting with $D=3, \mathcal{V}=\frac{1}{2} \oplus 0$, adding a spin-0 (increasing $D$ to 4 ) barely changes the variational energy, as seen in Fig. 5. However, adding a virtual spin-1/2 $(D=5)$ or a spin-1 $(D=6)$ does significantly improve the energy. We also note that the energy gain by adding the $\mathcal{B}_{1}$ components (therefore breaking the $\pi / 2$ lattice rotation symmetry) becomes larger with increasing $K_{1}$. This indicates that the nematic order becomes more prominent with increasing $K_{1}$ as discussed below.

Similar to energy density, the order parameter Eq. (2) associated with nematic PEPS Eq. (5), can also be calculated using CTM RG environment tensors, with the same setting as in Fig. 4(c). The results are shown in Fig. 6. Unlike energy, which monotonically decreases with increasing $D$, the order 


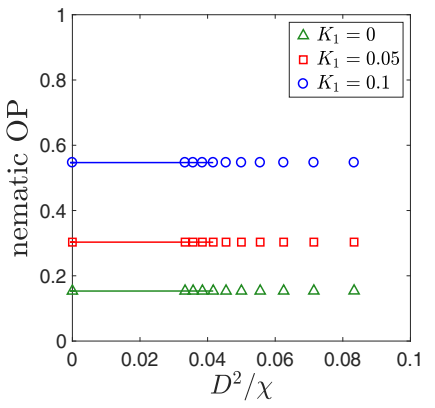

(a) $D=3$

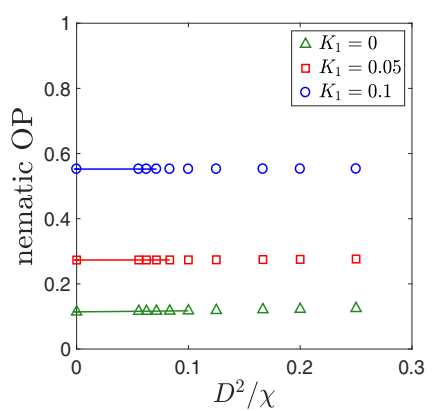

(c) $D=5$

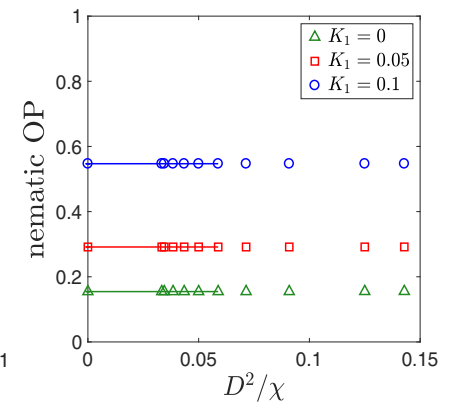

(b) $D=4$

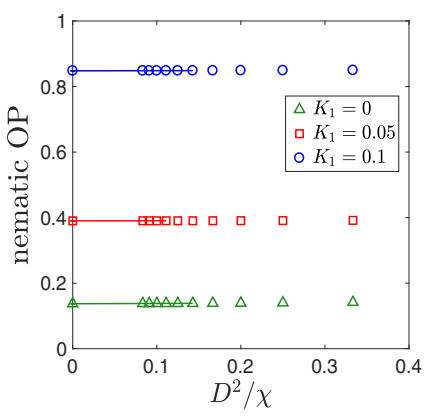

(d) $D=6$
FIG. 6. iPEPS nematic order parameter (2) for bond dimension $D$ from 3 to 6 , plotted as a function of $D^{2} / \chi$. The extrapolated value $(\chi \rightarrow \infty)$ is also shown, and the error bars are smaller than symbol sizes.

parameter does not behave smoothly with $D$. Nevertheless, we can see that, at $K_{1}=0$, the magnitude of the order almost shows no change with increasing $D$, whereas the changes are more significant for nonzero $K_{1}$, especially at $K_{1}=0.1$. More importantly, we find that, for each given bond dimension $D$, the order increases with $K_{1}$, showing full consistency with the increasing energy gain with respect to the symmetric $\mathcal{A}_{1}$ PEPS. This again shows that the $K_{1}$ term tends to stabilize the nematic phase.

We now explore further the physical properties of both the optimized nematic and symmetric PEPS wavefunctions by looking at the maximal correlation length $\xi_{\max }$, which can be obtained from the transfer matrix spectrum [31], as shown in Figs. 4(g) and 4(h):

$$
\xi_{\max }=-\frac{1}{\ln \left|\lambda_{2} / \lambda_{1}\right|}
$$

where $\lambda_{1,2}$ are the largest two eigenvalues of the effective transfer matrix $\mathcal{T}_{\text {eff }}$, which are symmetric matrices due to reflection symmetry. Note that, $\lambda_{1}$ is in general non-degenerate, and $\left|\lambda_{2}\right|$ is strictly smaller than $\left|\lambda_{1}\right|$, consistent with the absence of long-range order in each state. Since the horizontal and vertical directions are nonequivalent in a nematic state, we naturally have two different maximal correlation lengths $\xi_{\max }^{x}$ and $\xi_{\max }^{y}$.

The optimized fully symmetric $\mathcal{A}_{1}$ states are all critical with diverging correlation lengths, as revealed in Fig. 7(a) for $K_{1}=0$ and in Appendix $\mathrm{C}$ for other values of $K_{1}$, showing a linear increase of the correlation length $\xi_{\max }$ with environment bond dimension $\chi$, with no sign of saturation. The criticality of $D=3 \mathcal{A}_{1}$ state, i.e., RAL state, has been studied in Ref. [30], where it was found that, on the square lattice, the RAL state has exponentially decaying spin-spin correlation function but algebraically decaying dimer-dimer correlations. This is similar to the property of the NN RVB state [31], the criticality of both states being related to the exact U(1) gauge symmetry they possess. For the RAL state, this U(1) gauge symmetry is connected to the fact that there are always exactly two virtual dimers attached to every site. The criticality of the $D=4 \mathcal{A}_{1}$ state follows from the one of the RAL state, since only a spin- 0 is added to the $D=3$ virtual space and the property of having exactly two virtual dimers on every site is preserved. Therefore, it is also related to the same U(1) gauge symmetry. For the $D=5(D=6) \mathcal{A}_{1}$ states with virtual space $\mathcal{V}=\frac{1}{2} \oplus \frac{1}{2} \oplus 0\left(1 \oplus \frac{1}{2} \oplus 0\right)$, only the parity of the number of virtual dimers is a good quantum number, leading to a smaller $\mathbb{Z}_{2}$ gauge symmetry, from which one would (naively) expect a finite correlation length. However, the linear increase of the correlation length $\xi_{\max }$ with $\chi$ strongly suggests that these PEPS are also critical. These features are quite similar to the ones of the long-range RVB state [31], which bears both a discrete $\mathbb{Z}_{2}$ gauge symmetry and critical (dimer) correlations. It is likely that the $D=5,6 \mathcal{A}_{1}$ states also have emergent $\mathrm{U}(1)$ gauge symmetry, responsible for their critical nature.

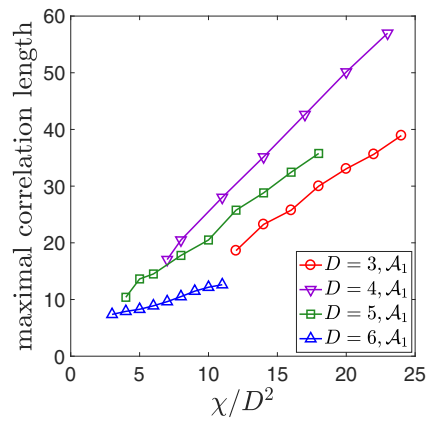

(a) $K_{1}=0$

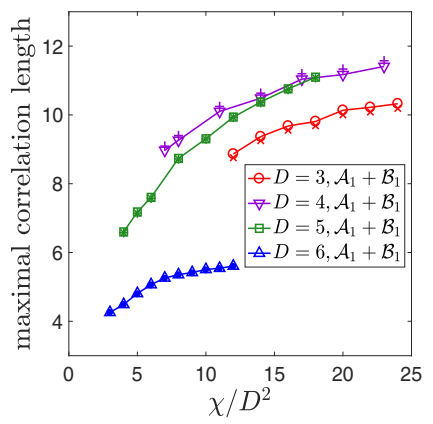

(b) $K_{1}=0$

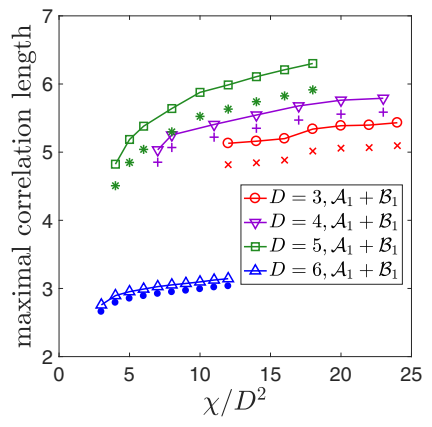

(c) $K_{1}=0.05$

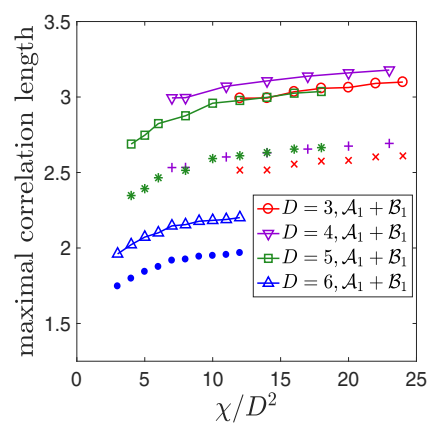

(d) $K_{1}=0.1$

FIG. 7. iPEPS maximal correlation length $\xi_{\max }$ in the (a) symmetric PEPS and (b)-(d) nematic PEPS vs $\chi / D^{2}$, for different values of $K_{1}$. The data for different values of $D$ ranging from 3 to 6 are shown with different symbols according to the legends. (a) and (b) $K_{1}=0$; (c) $K_{1}=0.05$; (d) $K_{1}=0.1$. In (b)-(d), open/filled symbols with same color correspond to the weak/strong bond directions of the nematic state. 
In deep contrast, the nematic states (Eq. (5)) all have finite correlation lengths, which clearly saturate with increasing $\chi$, as shown in Figs. 7(b) -7 (d) for $K_{1}=0,0.05,0.1$, respectively. This behavior is consistent with symmetry breaking, which lowers the energy by inducing a nonzero order parameter and would develop a gap (in the spectrum of any local parent Hamiltonian). The magnitude of the energy gain is proportional to the size of the order parameter and, approximately, to the size of the gap, which is itself inversely proportional to the correlation length. Thus, the decrease of the (saturated) correlation length with increasing $K_{1}$ (and the increase of the difference between $\xi_{\max }^{x}$ and $\xi_{\max }^{y}$ ) is consistent with: (i) the increase of the energy gain with respect to the symmetric (critical) PEPS and (ii) the increase of the nematic OP, as we can see in Figs. 5 and 6, respectively. This also indicates that $K_{1}=0$ is close to a critical point (even though the actual transition with the Néel state may be weakly first order), and that the nematic phase becomes more stable with increasing $K_{1}$, before the stripe magnetic order sets in abruptly. It is interesting to observe that, in contrast, $\xi_{\max }$ of the symmetric state diverges faster (see Appendix C) with increasing $K_{1}$. It is also worth mentioning that, even in the presence of an exact $U(1)$ gauge symmetry, the nematic state has finite correlation length, as we have checked for linear combinations of $D=3 \mathcal{A}_{1}$ and $\mathcal{B}_{1}$ tensors [32].

Finally, we note that, although the integer nature of the physical spin together with the spin-SU(2) symmetry formally leads to a $\mathbb{Z}_{2}$ gauge symmetry of the tensor (with the nontrivial group element generated by $2 \pi \mathrm{SU}(2)$ spin rotation [15]), we have checked explicitly using the tensor renormalization group method $[31,33,34]$ that the modular matrices of our optimized (short-ranged) nematic states are actually trivial, implying the absence of topological order in this system.

\section{SUMMARY AND OUTLOOK}

We have studied a nematic spin liquid phase in the frustrated spin-1 model with three complementary numerical methods: ED, DMRG, and iPEPS. Both ED and DMRG results suggest there exists a nonmagnetic phase which breaks $\pi / 2$ lattice rotation symmetry in the parameter region $0<K_{1}<0.15$, for fixed $J_{1}=1, J_{2}=0.54$. However, since ED can only deal with small system sizes and the cylinder geometry used in DMRG breaks lattice rotation symmetry, it is important to use a complementary method which: (i) works directly in the thermodynamic limit and (ii) enables to compare symmetric and symmetry-broken states. Hence, to get new insight on this plausible nematic phase, we have applied the iPEPS method to study the relevant parameter region directly in the thermodynamic limit.

Based on a previous classification of SU(2) spin rotation symmetric tensors [18], we systematically construct variational fully symmetric PEPS and nematic PEPS for the frustrated spin-1 Heisenberg model, which are all singlet states, targeting the correct nonmagnetic phase (without necessarily resorting to certain scaling with bond dimension $D$ ). The nematic PEPS is obtained by superposing two different classes of tensors, i.e., $\mathcal{A}_{1}$ and $\mathcal{B}_{1}$, while the fully symmetric PEPS is constructed only with $\mathcal{A}_{1}$ tensors. Through a comparison study with fully symmetric $\mathcal{A}_{1}$ PEPS and nematic $\mathcal{A}_{1}+\mathcal{B}_{1}$ PEPS, we unambiguously demonstrate the existence of the nematic phase. The PEPS variational energies agree very well with DMRG, therefore validating our ansatz. Through a detailed analysis of the nematic order parameter and the (maximal) correlation length of both symmetric and nematic PEPS, we find that the positive $\mathrm{NN}$ biquadratic term stabilizes the nematic phase, in agreement with ED and DMRG. It is interesting to note that the same nematic phase was also found with only NN bilinear and biquadratic terms on square lattice [35].

In summary, we have used a simple classification of SU(2) invariant PEPS to construct a generic family of well controlled ansätze of nematic spin liquids. The physical relevance of such states for a simple frustrated spin-1 Heisenberg model is established by direct comparisons to unbiased Lanczos ED and DMRG calculations. Such an approach could easily be extended to investigate many other types of lattice symmetry-breaking nonmagnetic phases in frustrated quantum spin models, and is left for future investigations.

Lastly, we point out that a possibly continuous transition between the $(\pi, \pi)$ Néel state and the nematic state can be described as a deconfined critical point of a noncompact $\mathrm{CP}^{1}$ model [36] which is conjectured to possess an emergent $O(4)$ symmetry [37,38]. However, our numerics cannot fully decide whether the transition is indeed continuous or weakly first order. Nevertheless, the symmetric critical $\mathcal{A}_{1}$ PEPS may provide a good representation of the critical point. Such an investigation is left for a future study.

Note added. Upon finishing this project, we became aware of related work [39].

\section{ACKNOWLEDGMENTS}

This project is supported by the TNSTRONG ANR Grant (French Research Council). This work was granted access to the HPC resources of CALMIP and GENCI supercomputing centers under the allocation No. 2017-P1231 and No. A0030500225, respectively. We acknowledge inspiring conversations with S.-S. Gong, P. Pujol, S. Sachdev, and D. N. Sheng. J.-Y.C. thanks H.-H. Tu for interesting discussion. We thank H.-C. Jiang for sending us the torus DMRG data for comparison, and thank Centro de Ciencias de Benasque Pedro Pascual for hospitality, where this work was finalized.

\section{APPENDIX A: ADDITIONAL DATA FROM DMRG SIMULATIONS}

\section{Convergence of energy and nematic OP}

As is usually done in DMRG, for each given $L \times W$ cylinder one can attempt to extrapolate ground-state total energy with respect to the discarded weight, see Fig. 8(a). In order to reduce the effects of open boundary conditions along the long direction $(L)$, it is also suitable to perform simulations on different cylinders of lengths $L_{1}$ and $L_{2}>L_{1}$ and obtain an estimate of the ground-state energy density using

$$
e_{0}(W)=\frac{E_{0}\left(L_{2}, W\right)-E_{0}\left(L_{1}, W\right)}{\left(L_{2}-L_{1}\right) W} .
$$

Note that when performing such extrapolations, the estimate is not variational anymore. We have used this procedure to get the best estimate of $e_{0}$ to be compared to iPEPS values. 

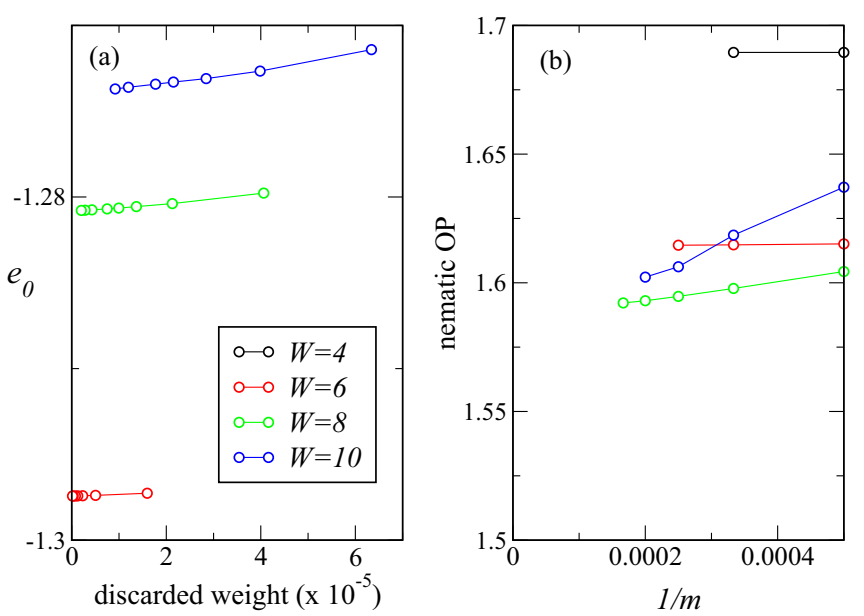

FIG. 8. Extrapolations performed for DMRG simulations on cylinder $2 W \times W$ at fixed $K_{1}=0.05$. (a) The ground-state energy per site $e_{0}$ is extrapolated with respect to the discarded weight. (b) The nematic order parameter measured in the bulk of the system is extrapolated with respect to $1 / \mathrm{m}$.

Regarding the nematic order parameter (2), for a fixed cylinder, we have chosen to extrapolate its value with respect to $1 / m$ where $m$ is the number of states kept in the DMRG (up to 6000 in our largest simulations), see Fig. 8(b). Moreover, for a fixed width $W$, we have checked that its value is rather independent on $L$ so that even shorter cylinders (i.e., $L<2 W$ ) could be used (data not shown).

\section{Bond energies on a finite cylinder}

By simply measuring the bilinear bond energies $\left(\left\langle\mathbf{S}_{i} \cdot \mathbf{S}_{j}\right\rangle\right.$ for neighboring $(i, j)$ sites), we can already visualize a qualitative change when $K_{1} \gtrsim 0$, see Fig. 9. In this range of parameters, the horizontal bonds become slightly ferromagnetic and quite different from the vertical ones, hence suggesting a possible nematicity. Nevertheless, a cautious analysis is needed since these data were obtained on cylinders (with an
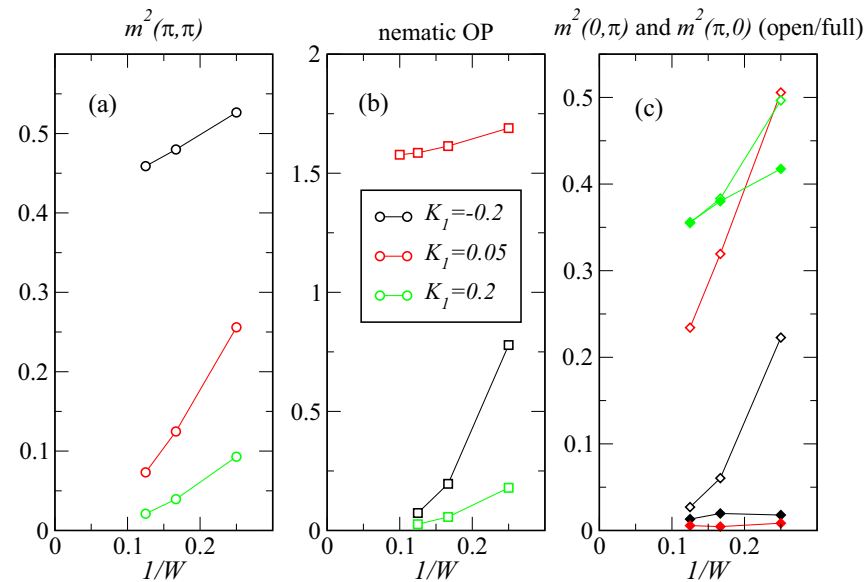

FIG. 10. Finite-size scaling of DMRG data obtained on cylinders $2 W \times W$ vs $1 / W$ for three typical values of $K_{1}=-0.2,0.05$, and 0.2 corresponding to the Néel, nematic, and stripe phases, respectively.

aspect ratio of 2) so that horizontal and vertical bonds are not equivalent, see a detailed analysis below and in the main text.

\section{Finite-size scaling of the order parameters}

As defined in the main text, we can compute several order parameters for the putative phases, but we do need to perform some finite-size scaling analysis. We have measured the spin structure factor and nematic order parameter in the bulk (i.e., in the center) of each $2 W \times W$ cylinder in order to reduce finite-size effects. In Fig. 10, we provide some scaling analysis vs $1 / W$. In particular, when $K_{1}=0.2$, the structure factor at wavevector $(0, \pi)$ is much stronger than $(\pi, 0)$ due to the cylinder geometry (see the pattern in real-space correlations in Fig. 2), but it is dominated by short-distance properties and is shown to extrapolate to the same value than $m^{2}(\pi, 0)$. Hence we choose this latter quantity as order parameter for the stripe phase.

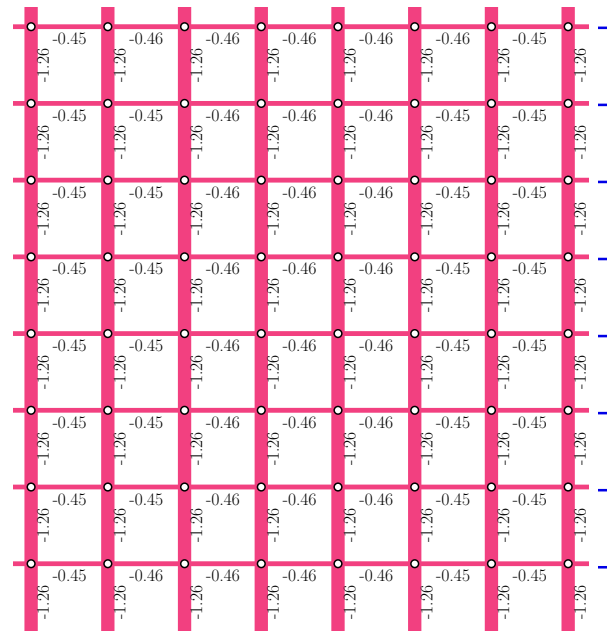

(a)

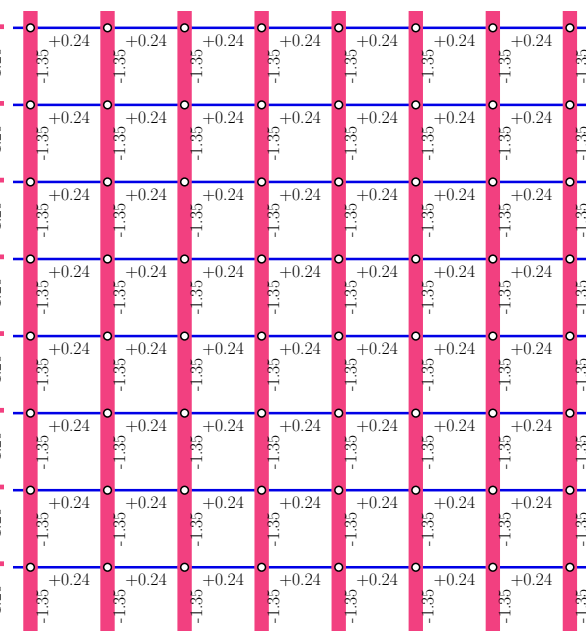

(b)

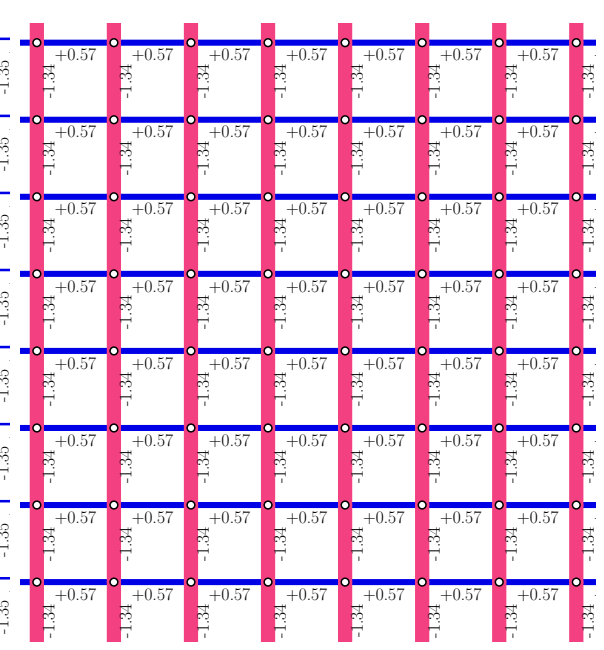

(c)

FIG. 9. Bond energies obtained by DMRG on a $16 \times 8$ cylinder keeping up to $m=5000$ states. (a) $K_{1}=0$. (b) $K_{1}=0.05$. (c) $K_{1}=0.1$. 


\section{APPENDIX B: SCALING OF THE IPEPS VARIATIONAL ENERGIES VS INVERSE ENVIRONMENT BOND DIMENSION}

PEPS variational energies are computed directly in the thermodynamic limit using an approximate contraction scheme

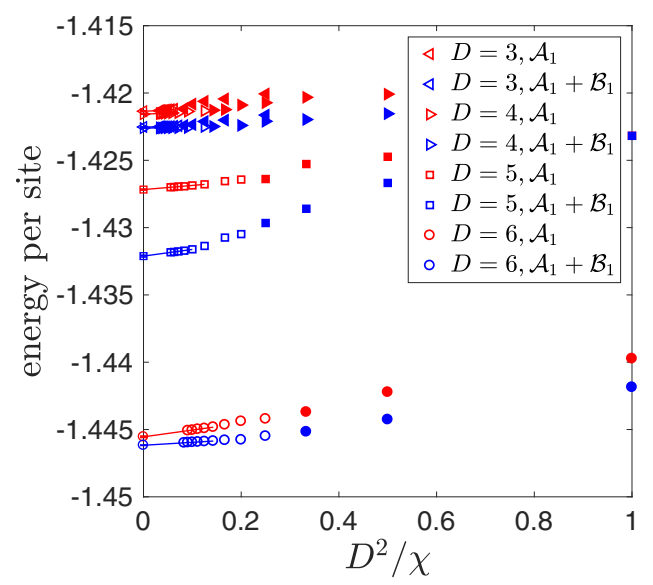

(a) $K_{1}=0$

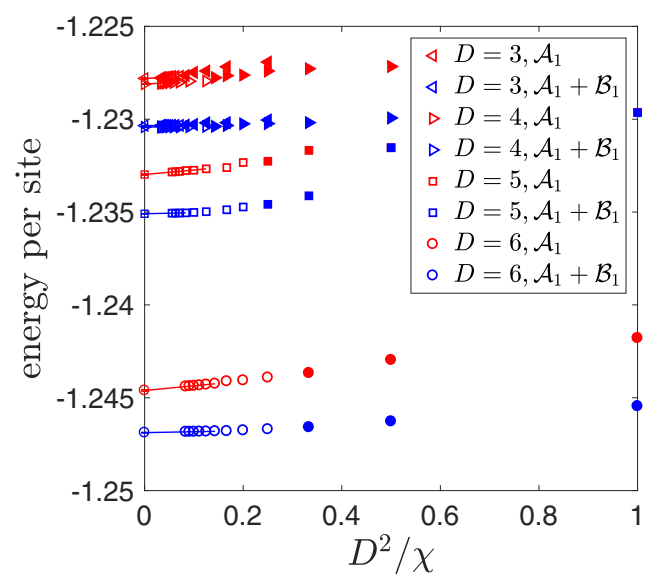

(b) $K_{1}=0.05$

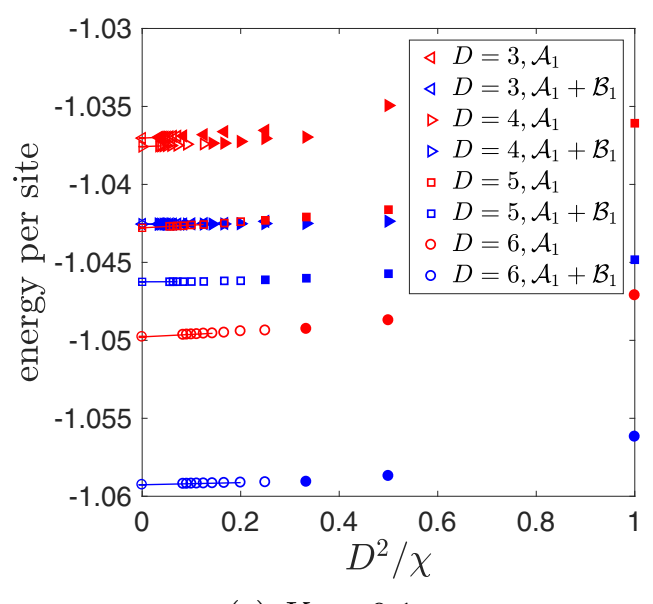

(c) $K_{1}=0.1$

FIG. 11. iPEPS energy density for various bond dimensions $D$, plotted as a function of $D^{2} / \chi$. (a) $K_{1}=0$. (b) $K_{1}=0.05$. (c) $K_{1}=0.1$. based on the CTM RG to obtain an effective environment with finite bond dimension $\chi$. The optimizations with respect to the tensor parameters $\left\{\lambda_{a}, \mu_{b}\right\}\left(\mathcal{A}_{1}+\mathcal{B}_{1}\right.$ classes $)$ or $\left\{\lambda_{a}\right\}\left(\mathcal{A}_{1}\right.$ classes $)$ have been performed up to $\chi=\chi_{\text {opt }}=$ $108,112,100,108$ for $D=3,4,5,6$, respectively (see main text). For larger $\chi$ a "frozen" ansatz obtained with $\chi=\chi_{\text {opt }}$ is used. Eventually an extrapolation to $\chi=\infty$ is performed by fitting the data plotted vs $D^{2} / \chi$, as shown in Fig. 11 .

Also, as is clear in Fig. 11, as we increase $\chi=k D^{2}(k \in$ $\mathbb{N}_{+}$), the energy goes down continuously. It is likely that this monotonous behavior applies to arbitrary large $\chi$ (although one cannot prove it), in which case the iPEPS energy can be safely considered as variational.

\section{APPENDIX C: SCALING OF THE MAXIMAL CORRELATION LENGTH VS ENVIRONMENT BOND DIMENSION}

Here, we present additional data of the maximal correlation length $\xi_{\max }$, shown in Fig. 12.

The trends that: (i) in the symmetric $\mathcal{A}_{1}$ PEPS, $\xi_{\text {max }}$ diverges faster with increasing $K_{1}$, whereas (ii) in the nematic $\mathcal{A}_{1}+$ $\mathcal{B}_{1}$ PEPS, $\xi_{\max }$ converges to smaller and smaller values, can be clearly seen in Fig. 12. (ii) shows full consistency with a discrete GL symmetry-breaking scenario.

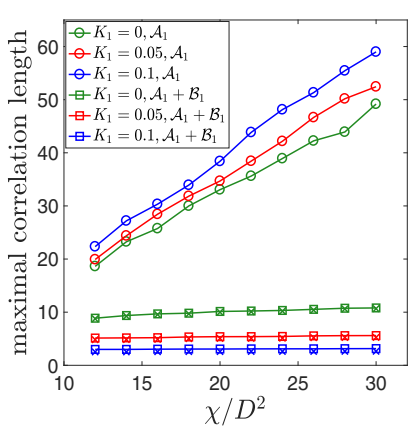

(a) $D=3$

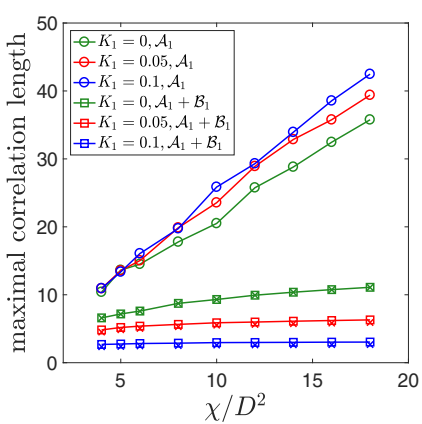

(c) $D=5$

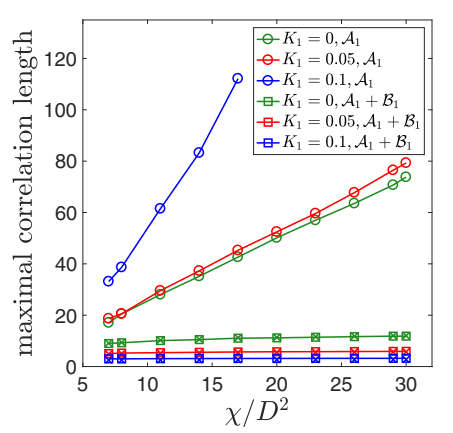

(b) $D=4$

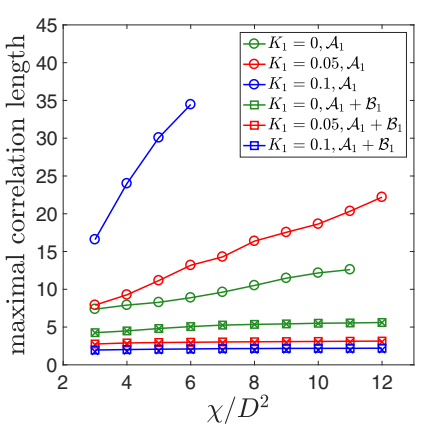

(d) $D=6$
FIG. 12. iPEPS correlation length $\xi_{\max }$ vs $\chi / D^{2}$. Each panel corresponds to a given value of the virtual dimension $D$. Open squares/crosses with same color correspond to the weak/strong bond directions of the nematic $\mathcal{A}_{1}+\mathcal{B}_{1}$ PEPS. Open circles correspond to the symmetric $\mathcal{A}_{1}$ PEPS. 
[1] T.-M. Chuang, M. P. Allan, J. Lee, Y. Xie, N. Ni, S. L. Bud'ko, G. S. Boebinger, P. C. Canfield, and J. C. Davis, Nematic electronic structure in the "parent" state of the iron-based superconductor $\mathrm{Ca}\left(\mathrm{Fe}_{1-x} \mathrm{Co}_{x}\right)_{2} \mathrm{As}_{2}$, Science 327, 181 (2010).

[2] E. Fradkin and S. A. Kivelson, Electron nematic phases proliferate, Science 327, 155 (2010).

[3] S. Ermakov, A. Beletskii, O. Eismont, and V. Nikolaev, Brief Review of Liquid Crystals, in Liquid Crystals in Biotribology: Synovial Joint Treatment (Springer, Cham, 2016), pp. 37-56.

[4] C. Weber, L. Capriotti, G. Misguich, F. Becca, M. Elhajal, and F. Mila, Ising Transition Driven by Frustration in a 2D Classical Model with Continuous Symmetry, Phys. Rev. Lett. 91, 177202 (2003).

[5] M. Schecter, O. F. Syljuåsen, and J. Paaske, Nematic Bond Theory of Heisenberg Helimagnets, Phys. Rev. Lett. 119, 157202 (2017).

[6] F. Wang, S. A. Kivelson, and D.-H. Lee, Nematicity and quantum paramagnetism in FeSe, Nat. Phys. 11, 959 (2015).

[7] F. D. M. Haldane, $\mathrm{O}(3)$ Nonlinear $\sigma$ Model and the Topological Distinction between Integer- and Half-Integer-Spin Antiferromagnets in Two Dimensions, Phys. Rev. Lett. 61, 1029 (1988).

[8] N. Read and S. Sachdev, Valence-Bond and Spin-Peierls Ground States of Low-Dimensional Quantum Antiferromagnets, Phys. Rev. Lett. 62, 1694 (1989).

[9] H. C. Jiang, F. Krüger, J. E. Moore, D. N. Sheng, J. Zaanen, and Z. Y. Weng, Phase diagram of the frustrated spatially-anisotropic $S=1$ antiferromagnet on a square lattice, Phys. Rev. B 79, 174409 (2009).

[10] J. I. Cirac and F. Verstraete, Renormalization and tensor product states in spin chains and lattices, J. Phys. A: Math. Theor. 42, 504004 (2009).

[11] J. I. Cirac, Entanglement in many-body quantum systems, in Many-Body Physics with Ultracold Atoms (Les Houches School, Les Houches, France, 2010).

[12] R. Orús, A practical introduction to tensor networks: Matrix product states and projected entangled pair states, Ann. Phys. (NY) 349, 117 (2014).

[13] N. Schuch, Condensed matter applications of entanglement theory, in Quantum Information Processing: Lecture Notes, Schriften des Forschungszentrums Jülich. Reihe Schlüsseltechnologien/Key Technologies, 44th IFF Spring School (David P. DiVincenzo, Forschungszentrum Jülich, 2013), p. 29.

[14] R. Orús, Advances on tensor network theory: symmetries, fermions, entanglement, and holography, Eur. Phys. J. B 87, 1 (2014).

[15] S. Jiang and Y. Ran, Symmetric tensor networks and practical simulation algorithms to sharply identify classes of quantum phases distinguishable by short-range physics, Phys. Rev. B 92, 104414 (2015).

[16] Note that we get qualitatively similar results if we measure the full bond energies including the biquadratic term.

[17] Note that, strictly speaking, the nematic order parameter may not identically vanish in the stripe phase.

[18] M. Mambrini, R. Orús, and D. Poilblanc, Systematic construction of spin liquids on the square lattice from tensor networks with SU(2) symmetry, Phys. Rev. B 94, 205124 (2016).
[19] We note that, in some cases, Eq. (5) with a subset of the elementary tensors does not produce a nematic state but instead a fully symmetric state, although the tensor $\mathcal{A}$ breaks lattice rotation symmetry. But in general, the state represented by Eq. (5) is a nematic state.

[20] D. Poilblanc and M. Mambrini, Quantum critical phase with infinite projected entangled paired states, Phys. Rev. B 96, 014414 (2017).

[21] D. Poilblanc, Investigation of the chiral antiferromagnetic Heisenberg model using projected entangled pair states, Phys. Rev. B 96, 121118 (2017).

[22] T. Nishino and K. Okunishi, Corner transfer matrix renormalization group method, J. Phys. Soc. Jpn. 65, 891 (1996).

[23] T. Nishino, Y. Hieida, K. Okunishi, N. Maeshima, Y. Akutsu, and A. Gendiar, Two-dimensional tensor product variational formulation, Prog. Theor. Phys. 105, 409 (2001).

[24] R. Orús and G. Vidal, Simulation of two-dimensional quantum systems on an infinite lattice revisited: Corner transfer matrix for tensor contraction, Phys. Rev. B 80, 094403 (2009).

[25] R. Orús, Exploring corner transfer matrices and corner tensors for the classical simulation of quantum lattice systems, Phys. Rev. B 85, 205117 (2012).

[26] P. Corboz, Variational optimization with infinite projected entangled-pair states, Phys. Rev. B 94, 035133 (2016).

[27] L. Vanderstraeten, J. Haegeman, P. Corboz, and F. Verstraete, Gradient methods for variational optimization of projected entangled-pair states, Phys. Rev. B 94, 155123 (2016).

[28] W.-Y. Liu, S.-J. Dong, Y.-J. Han, G.-C. Guo, and L. He, Gradient optimization of finite projected entangled pair states, Phys. Rev. B 95, 195154 (2017).

[29] W. H. Press, S. A. Teukolsky, W. P. Vetterling, and B. P. Flannery, Numerical Recipes (Cambridge University Press, New York, USA, 2007).

[30] W. Li, S. Yang, M. Cheng, Z.-X. Liu, and H.-H. Tu, Topology and criticality in the resonating Affleck-Kennedy-Lieb-Tasaki loop spin liquid states, Phys. Rev. B 89, 174411 (2014).

[31] J.-Y. Chen and D. Poilblanc, Topological $\mathbb{Z}_{2}$ resonating-valencebond spin liquid on the square lattice, Phys. Rev. B 97, 161107 (2018).

[32] The $D=3 \mathcal{A}_{1}$ class contains two tensors, both of which have two virtual spin- $1 / 2$ 's on every site configuration. The $D=3 \mathcal{B}_{1}$ class contains two tensors, denoted as $\mathcal{B}_{1}^{(1)}$ with two virtual spin-1/2's on every site configuration and $\mathcal{B}_{1}^{(2)}$ with four virtual spin-1/2's on every site configuration. The PEPS constructed by mixing $\mathcal{A}_{1}$ and $\mathcal{B}_{1}^{(1)}$ tensors has $\mathrm{U}(1)$ gauge symmetry but also a finite correlation length.

[33] H. He, H. Moradi, and X.-G. Wen, Modular matrices as topological order parameter by a gauge-symmetry-preserved tensor renormalization approach, Phys. Rev. B 90, 205114 (2014).

[34] J.-W. Mei, J.-Y. Chen, H. He, and X.-G. Wen, Gapped spin liquid with $\mathbb{Z}_{2}$ topological order for the kagome Heisenberg model, Phys. Rev. B 95, 235107 (2017).

[35] I. Niesen and P. Corboz, Emergent Haldane phase in the $s=1$ bilinear-biquadratic Heisenberg model on the square lattice, Phys. Rev. B 95, 180404 (2017).

[36] T. Senthil, A. Vishwanath, L. Balents, S. Sachdev, and M. P. A. Fisher, Deconfined quantum critical points, Science 303, 1490 (2004). 
[37] M. A. Metlitski and R. Thorngren, Intrinsic and emergent anomalies at deconfined critical points, arXiv:1707.07686.

[38] C. Wang, A. Nahum, M. A. Metlitski, C. Xu, and T. Senthil, Deconfined Quantum Critical Points: Symmetries and Dualities, Phys. Rev. X 7, 031051 (2017).
[39] R. Haghshenas, W.-W. Lan, S.-S. Gong, and D. N. Sheng, Quantum phase diagram of spin-1 $J_{1}-J_{2}$ Heisenberg model on the square lattice: An infinite projected entangled-pair state and density matrix renormalization group study, Phys. Rev. B 97, 184436 (2018). 